\title{
Self-Tracking for Health and the Quantified Self: Re-Articulating Autonomy, Solidarity, and Authenticity in an Age of Personalized Healthcare
}

\author{
$\operatorname{Tamar}_{\text {Sharon }}{ }^{(D)}$
}

Received: 30 September 2015 / Accepted: 16 March 2016 / Published online: 18 April 2016

(C) The Author(s) 2016. This article is published with open access at Springerlink.com

\begin{abstract}
Self-tracking devices point to a future in which individuals will be more involved in the management of their health and will generate data that will benefit clinical decision making and research. They have thus attracted enthusiasm from medical and public health professionals as key players in the move toward participatory and personalized healthcare. Critics, however, have begun to articulate a number of broader societal and ethical concerns regarding self-tracking, foregrounding their disciplining, and disempowering effects. This paper has two aims: first, to analyze some of the key promises and concerns that inform this polarized debate. I argue that far from being solely about health outcomes, this debate is very much about fundamental values that are at stake in the move toward personalized healthcare, namely, the values of autonomy, solidarity, and authenticity. The second aim is to provide a framework within which an alternative approach to self-tracking for health can be developed. I suggest that a practice-based approach, which studies how values are enacted in specific practices, can open the way for a new set of theoretical questions. In the last part of the paper, I sketch out how this can work by describing various enactments of autonomy, solidarity, and authenticity among self-trackers in the Quantified Self community. These examples show that shifting attention to practices can render visible alternative and sometimes unexpected enactments of values. Insofar as these may challenge both the promises and concerns in the debate on self-tracking for health, they can lay the groundwork for new conceptual interventions in future research.
\end{abstract}

Keywords Self-tracking · Digital health $\cdot$ Quantified self $\cdot$ Surveillance $\cdot$ Practice-based approach $\cdot$ Personalized healthcare $\cdot$ Philosophy of technology

Tamar Sharon

tamar.sharon@maastrichtuniversity.nl

1 Faculty of Arts and Social Sciences, Philosophy Department, Maastricht University, Postbus 616, 6200 MD Maastricht, Netherlands 


\section{Introduction}

In less than a decade, a steady stream of new smartphone applications and wearable mobile sensors that allow users to monitor sleep, food intake, exercise, blood sugar, mood, and a host of other physiological states and behaviors has permeated the consumer health landscape, bearing the promise of cheaper, better, and more efficient healthcare. Self-tracking devices point to a future in which individuals will be more involved in the management of their own health and will generate health information that will benefit clinical decision making and research. Today, references to disruptive paradigm shifts and imminent revolutions saturate popular media and policy reports, as these technologies draw growing support from medical professionals, public health officials, and funding agencies (Topol 2015; EC 2012, 2014a; Steinhubl et al. 2013; NHS 2015), and become increasingly popular among individual consumers. As one enthusiast has put it, "the paradigm of the old thinking 'My health is the responsibility of my physician', [is] being replaced by the new thinking that 'My health is my responsibility, and I have the tools to manage it" " (Swan 2012, p. 108). Self-tracking for health ${ }^{1}$ is thus expected to play a key role in the move toward "personalized healthcare," the model of targeted, preventive, and participatory healthcare that is being envisioned as a solution to the crisis of public healthcare systems in the industrialized world (Norris 2012; Flores et al. 2013). Recently, in response to the overwhelmingly positive attention that self-tracking has received in the medical and public health literature, critical discussions of self-tracking for health have begun to emerge in the social sciences literature (e.g., Lupton 2012, 2013, 2014, 2015; Morozov 2013; Whitson 2013; Nafus and Sherman 2014; Ruckenstein 2014; Till 2014). These critical analyses articulate a number of concerns regarding the social, cultural, political, and ethical implications of self-tracking and the move toward more personalized healthcare.

This paper has two aims: to analyze some of the key polarizations that structure this debate, which increasingly will act as a backdrop for decision making concerning the integration and regulation of self-tracking devices in the healthcare context and beyond, and, further, to provide a framework within which an alternative approach on self-tracking for health can be developed, by reframing the debate on self-tracking for health in terms of values that are enacted in self-tracking practices. In the first part of the paper, I offer an extensive analysis of the discussions on self-tracking for health based on the promises and concerns that are being voiced in policy reports, medical and public health literature, industry reports, advertising, and critical social science literature. I organize this analysis around three main promises and concerns: empowerment vs. surveillance and discipline, improved overall health vs. the disintegration of state and collective responsibility for health, and greater (self-)knowledge vs. reductionism and the non-impartiality of numbers.

Next, I argue that while these promises and concerns need to be taken seriously, several issues point to the need to move beyond them, namely, insufficient empirical support and problematic configurations of individual users, which make this debate somewhat detached from the everyday experiences of users of self-tracking devices. Furthermore, I argue that far from being solely about health outcomes, the promises and concerns that inform this debate should be understood as normative positions relating

\footnotetext{
${ }^{1}$ In this article, I use the term "self-tracking for health" where the terms "mobile health," "mHealth," and "digital health" are also common.
} 
to three fundamental values that are at stake in the move toward personalized healthcare: autonomy, solidarity, and authenticity. I suggest that a practice-based approach (Mol 2002; Swierstra 2013), which studies how values are articulated or enacted in specific practices, is useful for moving beyond these limitations and can open the way for a new set of theoretical questions. In the last part, I sketch out how this can work by describing various enactments of autonomy, solidarity, and authenticity in the self-tracking practices of the Quantified Self community, a large group of avid selftrackers. These examples show that when we re-orient our attention to practices, alternative and sometimes unexpected enactments of values are made visible. Insofar as these may challenge the problematic discourses of empowerment and autonomous choice that inform the promises of self-tracking for health, but also indicate that the concerns involved may be unwarranted, they can lay the groundwork for new conceptual interventions in future research.

\section{Self-Tracking for Health: Prime Mover of the Personalized Healthcare Revolution}

In recent decades, spiraling costs, an ageing population, and the rise of chronic diseases have propelled public healthcare systems in the industrialized West into crisis. For many policy makers and health professionals, the solution resides in a new vision of personalized medicine and healthcare: a move from a "one-size-fits-all" model, to healthcare that is preventive, participatory, and tailored to the specific characteristics of individuals (Coggi 2011; Byrne and Brand 2013; EC 2013; ESF 2012). Two fundamental components of personalized healthcare (PHC), the combination of which underlies perhaps more than anything else the revolutionary claims of its proponents, are its emphasis on data and its emphasis on individuals. PHC is a data-rich endeavor. The integration of vast amounts of heterogeneous biological, environmental, and lifestyle data lies at the core of its ambitions to target, tailor, and personalize care to each individual's unique biological makeup and health experience (NAS 2011; Cesuroglo et al. 2012). Advocates of PHC also maintain that it is revolutionary insofar as it is citizen- or patient-centered. Patients and citizens are constituted as both the main contributors to and the main beneficiaries of PHC. Personalized health information, it is claimed, should enable individuals to become more informed, more engaged, and more in control of their health. This active, participatory role of citizens and patients is vital. Not just because it is hoped that an increased health responsibilization on the part of individuals will reduce healthcare spending, but because $\mathrm{PHC}$ - as a data-driven approach - requires the active participation of citizens as generators and contributors of data (ESF 2012).

These two essential aspects of PHC, its data-centeredness and its participatory form, are brought together in self-tracking devices, wearable sensors, and mobile applications that allow users to collect, measure, and display data concerning virtually any form of bodily function and behavioral activity. These include thousands of health-related apps for smartphones and tablets that can track information about food consumption, sleep patterns, blood chemistry, moods, menstrual cycles, heart rate, and stress levels. Popular mobile health apps for weight loss and management such as "MyFitnessPal" or "DailyBurn," for example, allow 
users to enter details of their meals and monitor their progress toward a desired weight. Apps directed at managing various chronic conditions including diabetes, Crohn's disease, asthma, heart conditions, and chronic pain act like digital journals, allowing users to $\log$ symptoms, the effectiveness of treatment and medications, and reactions to different foods and environments. These apps usually offer an analysis of the recorded data in graph format, so users can visualize trends, such as when asthma symptoms peak, or what triggers high blood pressure. Mental well-being is another popular area for self-tracking. The "Moodscope" app, for example, allows users to score and track their daily moods and share these with a nominated "buddy." Other devices are designed to be worn directly on the body, automatically collecting data and wirelessly syncing it to the user's computer or smartphone. The popular Fitbit, Jawbone, or Nike Fuelband, for example, measure steps made, stairs climbed, and calories burned, transform the data into graphs and charts, and analyze it. Typically, this data can be uploaded to related websites and social media platforms where it can be compared and shared with friends, while various gaming and reward mechanisms aim to keep users motivated. More recently, a range of sensor-embedded clothing, jewelry, adhesive patches, and smart "objects" (forks that detect how fast a user eats, baby bottles that monitor milk consumption, jars that communicate nutritional value of contents) have also been unveiled.

The hope with all of these devices is that they will enable people to achieve healthier habits and lifestyles and to better manage chronic conditions. Of course, the idea of keeping track of bodily processes and emotional states in the aim of some form of self-improvement is not new. Athletes, people with chronic conditions, and anyone who has systematically used a scale to watch their weight have all practiced, to some degree, self-tracking. But the advent of inexpensive digital self-tracking tools, which have made the collection and analysis of data easier, more precise and, arguably, more fun, has led to a dizzying rise in the phenomenon of self-tracking for health. A Pew Internet study conducted in 2013 found that 1 in 5 Americans use some form of technology to track their health (Fox and Duggan 2013), and it is estimated that by 2017, almost 2 billion people worldwide will use digital devices to track their health (Research2Guidance 2013). Currently, some 97,000 mobile health apps exist, and an estimated 485 million wearable devices are expected to be released annually by 2018 (ABI 2013).

In light of this growing popularity and given its purported potential for both generating valuable health data and enabling citizens to participate in their own care, self-tracking for health is expected to play a significant role in the development and implementation of PHC. European institutions and national governments are showing widespread support for the integration of digital and mobile health tools into healthcare (NHS 2011, 2015; EC 2012, 2014a, b, c), and an array of medical and public health professionals have begun to express enthusiasm for how self-tracking devices can be effectively used as tools for helping people deal with medical conditions and improving public health (Harrison et al. 2011; Laakso et al. 2011; Steinhubl et al. 2013). Indeed, as per some of the most out-spoken advocates of mobile health, self-tracking technologies seem to have thrown us onto the cusp of "yet another revolution in healthcare, brought to you by the patient herself as she uses her phone for self-tracking" (Wiederhold 2012). 


\section{A Polarized Debate}

Not everyone is enthusiastic about the prospects of a widespread adoption of selftracking for health, nor of the more general move toward PHC, however. In response to the overwhelmingly positive attention that self-tracking has received in the medical and public health literature, critical discussions of self-tracking for health have begun to emerge in the social sciences literature (e.g., Lupton 2012, 2013, 2014, 2015; Morozov 2013; Whitson 2013; Nafus and Sherman 2014; Ruckenstein 2014; Till 2014), giving rise to a broad, polarized debate. In the following, I analyze three important polarizations involved in this debate that it is helpful to look at in some detail.

\subsection{Empowerment vs. Surveillance and Discipline}

The promise of empowerment resonates widely across the discursive landscape of selftracking for health. Empowerment terminology in healthcare is not, in itself, new. Over the past few decades, it has come to replace a terminology of compliance in care and paternalistic or authoritarian models of the patient-doctor relationship (Traynor 2003), while becoming the hallmark of medical ethics in healthcare law. The novelty with selftracking for health is that it portrays empowerment as one of its paramount virtues. As mentioned, very much in line with the hype of PHC, what is seen as revolutionary with the advent of mobile health is not just the type of medicine and care that will be made possible (more targeted, more preventive), but that it will allow ordinary people to take more control over their health.

Advertisements and websites for wearables and apps typically portray lifestyle and chronic conditions as unruly things that need to be taken charge over: users are called upon to "take control of your sleep," "eat smarter," and "tell your weight who's boss," and devices are depicted as facilitators that can "help you on your quest [toward glucose control]" (www.glucosebuddy.com). At the same time, the data that is generated is often portrayed as knowledge that will enable users to have more informed discussions with their healthcare practitioner, thus rectifying what is presented as a traditionally asymmetrical relationship. Eric Topol, for example, one of the leading advocates of digital health, views the "democratization" of medicine as the most significant impact of the use of mobile devices in healthcare. In his latest book, Topol (2015) describes the widespread integration of mobile health devices as medicine's "Gutenberg moment," comparing the newfound control of patients over their care to the liberation of knowledge from the control of an elite class by the printing press. Empowerment as democratization is also emphasized by Melanie Swan, another outspoken visionary of PHC. "The role of the patient is starting to shift," she writes, "from being a minimally informed advice recipient to an active participant, instigating collaborator, information sharer, peer leader and self-tracker engaged in participative medicine; a transition is underway from paternalistic health care to partnership models" (2009, p, 513).

Similar messages concerning a technologically enabled passage from passive patients to active citizens are commonplace in the biomedical and public health discourse surrounding self-tracking for health and PHC. The P4 Medicine Institute boasts that "the passive patient will be transformed into the engaged consumer who takes ownership of his or her own health" (https://www.facebook.com/p4Mi.USA/info?tab=page_ 
info). The European Commission, in its Green Paper on mobile health, states that "mHealth solutions support the changing role of patients from a rather passive, to a more participative role" and that they can "contribute to the empowerment of patients as they could manage their health more actively, living more independent lives" (EC 2014a, pp. 5 and 3). In a press release on mobile health, the EC also places "put[ting] the patient in control" and "giving greater independence" at the top of the list of opportunities afforded by mobile health (2014c). Empowerment thus has several meanings for the various actors and stakeholder groups in the digital and personalized healthcare domain, including the following: gaining some control over erratic phenomena like habits and disease, a democratization of the doctor-patient relationship, increased personal responsibility over health, and allowing people to lead more independent lives by forestalling various forms of inpatient care.

The promise of patient empowerment has not gone unquestioned in critical studies of health. Here, empowerment narratives have often been understood as strategies of contemporary health promotion that encourage individuals to view their health as a project to be managed in a way that aligns with medical regimes, thus extending the reach of medical power (Burrows et al. 1995; Harris et al. 2010). In the context of selftracking for health, this argument gains new impetus. Mobile wireless devices that can collect biometric data anywhere and anytime and communicate it automatically to medical professionals wirelessly seem to offer an unprecedented opportunity on the part of public health officials to monitor and discipline people's health and lifestyle behaviors, not just of patients', but, in the aim of prevention, also those of healthy individuals. Self-tracking tools indeed entail a minimal level of commitment to selfexamination, self-monitoring, and self-improvement, placing them within a long history of medical surveillance and biopolitical governance (Foucault 1977; Armstrong 1995). The sociologist of health Deborah Lupton, who has undertaken an extended critical analysis of mobile health and self-tracking technologies in the last few years, writes that

The use of $\mathrm{m}$-health in health promotion extends the temporal nature of health surveillance, and allows for further refinements of the categorizing and identifying of "risk factors" and "at-risk groups" that are then deemed eligible for targeting. (Lupton 2012, p. 234)

Self-tracking for health, in such accounts, is portrayed as a paradigmatic practice of contemporary surveillance society, as one of the many ubiquitous technologies used to monitor, measure, and record individuals' activities for purposes of disciplining and managing populations via an extension of the medical or "panoptic" gaze. Yet, while surveillance has traditionally been conceptualized as something externally imposed, as Lupton argues, self-tracking gives rise to even more complex forms of monitoring, blurring the boundaries between private and public surveillance. Here, what she calls the "net of surveillance" is extended by encouraging users to turn the medical gaze upon themselves in the form of "self-surveillance" and to invite peers to participate in monitoring practices via the sharing of personal data on social media and other digital platforms. In addition, the "gamification" of self-tracking for health on these platforms, where self-trackers can compare their data against others', inviting ever further scrutiny, is perceived as leading to a greater normalization of intrusive surveillance practices. 
The positioning of self-tracking tools as part of a culture of surveillance implies that far from empowering individuals to be more in control, self-tracking for health is disempowering, insofar as it invites an increased control of others - health promoters, friends and followers, and even the internalized health promoter of one's own super ego- over oneself. For critical scholars of self-tracking, the tangible effects of this type of self-discipline and governmentality can already be seen, for example, in the incorporation of health-tracking practices in the workplace, as part of corporate "wellness programs" that offer lower health insurance premiums to employees based on steps counted using activity trackers (McGregor 2014; Olson 2014a, b). ${ }^{2}$ As critics remark, tracking devices offer many new ways of monitoring worker productivity throughout the day and in different environments, that may extend to tracking respiration, focus and stress levels. While employers usually explain that such programs aim to optimize the work place by making people healthier and adding a little "friendly competition" to improve morale and productivity, for critics, their disciplining effects are unmistakable (Lupton 2014; Till 2014). As they argue, the line between voluntary participation and compulsory participation is not always clear-cut.

Penalizing unhealthy behavior in the workplace in the form of more expensive premiums is just one clear example of the disempowering effects that greater health monitoring can have. Much of the surveillance literature also emphasizes how data generation practices that begin as personal and private become enmeshed in larger networks and economies without users being aware of this (boyd and Crawford 2012; Andrejevic 2014). Post-Snowden, it has become clear that the "data traces" left by unassuming individuals are of great value, for both national security agencies and for corporations. Chris Till (2014), for example, has recently analyzed some of the ways that large corporations manufacturing self-tracking devices like Nike and Fitbit are working to transform the data that their users generate-for free-into commercial value, by "sharing" this information with various third parties. Till and others draw on notions of "digital labor" or "free labor" (Terranova 2000) to indicate the new forms of unpaid labor that people carry out online under the guise of "fun" and "leisure" and that are highly profitable for these corporations. Along with the disciplinary effects of self-tracking and the idea that some types of self-tracking may become compulsory, these concerns greatly problematize the promise of empowerment put forward by advocates of self-tracking.

\subsection{Improved Overall Health vs. The Disintegration of State and Collective Responsibility for Health}

The second promise of self-tracking for health is improved overall health and more sustainable healthcare. For most stakeholders involved in PHC, there is an acknowledgment that the financial strain that healthcare systems in the industrialized West are facing is not only the result of the economic and financial crisis, but also largely due to

\footnotetext{
${ }^{2}$ ABI Research (2013) has found that more than 13 million wearable devices will be integrated into corporate wellness programs in the coming years. Such partnerships are common mainly in the United States today, where employers pay for the health insurance coverage of their employees. But, European insurance groups have also begun exploring the potential for policies that reward people based on information provided by tracking devices in cars and homes (De Corde and Rolvink Couzy 2015).
} 
what is seen as the ineffectiveness of the traditional model of reactive, one-size-fits-all medicine. PHC is thus a stone that kills two birds: its effectiveness is tantamount to its cost-efficiency. References to the need to cut spending and increase healthcare savings are thus never far off when the health benefits of PHC are being sung. In its Green Paper, the EC cites actual saving forecasts that mHealth can generate for EU healthcare by 2017: $€ 69$ billion in the area of wellness/prevention and $€ 32$ in treatment/monitoring - a total of $€ 99$ billion considering the costs of the workforce needed to support mHealth (EC 2014a, p. 6).

The improved overall health that personalized and mobile healthcare promise to deliver comprises many ingredients, including more precisely calibrated health interventions, diagnoses and treatments, fewer side effects, extended life expectancy, the reduction of unnecessary consultations, improved quality of life and patient comfort, better planning, better prepared professionals, and increased treatment rates (EAPM 2012; EC 2014a). One of the biggest novelties of digital self-tracking is that it makes it possible to collect data on individuals continuously, creating a much more complex and complete picture than if health data is only collected at regular intervals. Medical professionals have described the use of digital devices with reference to the successful management of conditions like hypertension, diabetes, mental health, and obesity, to improving psychological well-being, health behavior, and physical activity, and to reducing health risk factors (Harrison et al. 2011; Foreman et al. 2011; Steinhubl et al. 2013; Rabin and Bock 2011). A recent study by the Switzerland-based Soreon Research group predicted that wearable health tracking devices will save 1.3 million lives by 2020 (Soreon 2014).

Interestingly, the promise of improved and sustainable overall health here is presented as working in a bottom-up fashion that is not the traditional proposed mechanism for achieving population or public health goals, certainly not in the European context. There is an individualizing moment in the promissory discourse of selftracking for health and PHC - expressed in the very terms of "personalization" and "self-tracking" - that is crucial to the entire enterprise. Not just in terms of the appreciation of the uniqueness of each individual's health profile and the attempt to individualize treatments accordingly, but in the idea that the overall health (physical and financial) of the population can only be improved if individuals take on more personal responsibility for their own health. PHC and self-tracking for health echo the discourse of individual responsibility that has become pervasive in government policy on healthcare in the United States and in Europe in recent decades. Here, an increased emphasis on individual responsibility for health and healthy lifestyles as an important contribution to diminishing the burden of disease and financial costs has become explicit (ter Meulen and Jotterand 2008). In this sense, to be engaged in one's health is, ipso facto, to be engaged in the health of the population. Indeed, this is why the empowerment of individual patients and citizens is key.

This intertwining of the personal and collective good is particularly noticeable in the data-intensive endeavors of personalized and mobile health. As mentioned, the development and implementation of PHC require vast amounts of individualized health, environmental, and lifestyle data. The growing use of self-tracking devices means that, potentially, a significant amount of that data can be generated "beyond the clinic" (Prainsack and Vayena 2013) by patients and citizens themselves, thus reconfiguring them as knowledge producers, not 
just knowledge recipients (ESF 2012). In this way, citizens and patients are not just helping to improve population health by becoming more involved in their own health through self-monitoring and self-care; they can also actively contribute to medical decision making and research in ways that were inconceivable in earlier healthcare models.

Yet, since its emergence, the increased emphasis on individual responsibility in health has been the focus of intense critique by scholars working on health and governance issues. Here, the expectation that citizens play a more active role in caring for themselves is seen as replacing the notion that the state should care for the health of its citizens, on the backdrop of a withdrawal of state funding for social support and healthcare programs. Increased individual responsibility for health is thus perceived as emblematic of the transformation of the relationship between citizens and the state in a wider shift from welfare to neoliberal models of governance in advanced liberal societies (Burrows et al. 1995; Rose 1999). In the area of healthcare, as in other areas, the emphasis on individual responsibility is seen as seeking to transform citizens from passive recipients of state assistance into active, entrepreneurial, and "healthy citizens," who take responsibility for managing their health risks and have a moral commitment to pursuing a healthy lifestyle (Crawford 1980), while de-collectivizing public healthcare (Clarke et al. 2007).

Increased individual responsibility for health, then, is perceived not so much as empowering patients and citizens, but as above all directing the management of health away from the state and onto the shoulders of individual citizens. In a recent attack on the claims of PHC, Donna Dickenson (2013) suggests that economic and political factors shaped by neoliberal policies, rather than scientific plausibility, are what drive the move toward PHC - what she aptly calls a move from "We Medicine" to "Me Medicine." Dickenson argues that what characterizes PHC above all is the undermining of ideals of public health and the common good by rampant consumerism and individualism. "Me Medicine," she writes, "is typically portrayed as empowering, but the real power and legal rights rest with the corporate interests in this case, particularly when they're backed up by neoliberal government policy" (2013, pp. 23-24).

In this context, the use of self-tracking devices has also been conceptualized as promoting extreme forms of healthism and individualism. Lupton claims that selftracking devices represent the "apotheosis of self-reflexivity" (2013, p. 5). Selftrackers, she writes, are individuals who have "readily adopted the subject of the responsible, entrepreneurial citizen as it is privileged in neoliberal governmentality in seeking to take action to achieve a healthy and fit embodiment and engaging in selfgovernance" (2013, p. 6). Other accounts are less forgiving, describing self-trackers as a perverse product of this individualist mindset. Morozov writes " $[\mathrm{t}]$ he appeal of selftracking can only be understood when viewed against the modern narcissistic quest for uniqueness and exceptionalism" (2013, p. 233). Narcissism, "extreme navel-gazing" and "self-obsession" are terms that are not uncommon in depictions of self-trackers in the popular media (e.g., Hill 2011).

Critics also argue that, as a public health strategy, it is not at all clear that empowerment and individualization actually yield positive health benefits. What the individualistic approach to health does in this view is detach health from its broader social, political, and cultural dimensions, directing collective attention away from health 
threats posed by social or environmental conditions (Crawford 2006; Tutton 2012; Ayo 2012). Furthermore, when health is construed as a choice, those who do not, or cannot, "choose" health can be blamed for poor health, in forms ranging from public disdain and stigmatization for adding to the "burden" placed on the public health system, to penalizations or a withholding of treatments based on "lifestyle rationing" (e.g., Campbell 2012). In terms of the promise of improved overall health, this means that discourses of empowerment and individual choice are rhetorical devices that conceal political and economic agendas that are often detached from the real interests of patients and citizens, masking a dynamic of abandonment rather than empowerment.

\subsection{Greater (Self-)Knowledge vs. Reductionism and the Non-Impartiality of Numbers}

The third promise of self-tracking for health is greater knowledge: knowledge that will be more precise, more actionable, and that will cover ever more areas, including one's knowledge of oneself. One of the important novelties of PHC, as indicated above, is the centrality of data, not just genomic and other 'omics data, but also non-traditional sources of biomedical data like lifestyle and environmental data that are generated outside of the healthcare system. These vast heterogeneous datasets, or "big biomedical data," and the attempt to harness value from their large-scale integration and analysis herald exciting possibilities in the healthcare context (Groves et al. 2013) and a possible shift in epistemic criteria for what might be acceptable as "knowledge" (Dhar 2014). Linking big biomedical data, it is argued, will enable researchers to identify which treatments are most effective for particular conditions, to test new hypotheses and identify patterns related to drug side effects and to create the possibility of larger study populations than ever thought possible. "These data," write the authors of the EC Green Paper on mobile health,

can be a vital element of epidemiological research as they can enable scientists to improve patient treatment by looking for patterns on a larger scale or draw new conclusions, for instance on the relation between the development of a medical condition and environmental factors. (2014a, p. 9)

Moreover, it is argued that linking health data further with other sources, like social media, credit card purchases, and census records, will "help assemble a holistic view of a patient" (Weber et al. 2014). Self-tracking devices are portrayed as powerful tools in this radical, data-intensive rehaul of health research, as generators of the "small data" on any one individual user that will feed these vast datasets.

The University of San Diego, for example, has recently launched a "Health Data Exploration Project" that seeks to harness the potential of health-tracking devices. As per the project's website, "Self-tracking data can provide better measures of everyday behavior and lifestyle and can fill in gaps in more traditional clinical or public health data collection, giving us a more complete picture of health" (HDE 2016). Research projects using data generated by tracking devices are being initiated on various scales and in various formats. These include a growing number of joint private and public initiatives, such as collaborations between Apple and leading research centers using the ResearchKit platform, which allows clinicians to develop apps for carrying out medical 
studies on iPhones ${ }^{3}$, Google's "Baseline Study" in partnership with Duke and Stanford Universities, or the Institute for Systems Biology's "Hundred Person Wellness Project." Patient- or citizen-run studies using data generated by tracking and monitoring devices conducted individually or in groups are also becoming an important trend. Of note are studies organized by health social networks like PatientsLikeMe, which famously published a study analyzing the effect of lithium carbonate on ALS progression based on the self-reported data of its own members (Wicks et al. 2011). For a number of enthusiasts, the potential of such initiatives to accelerate clinical discovery, generate data, monitor long-term outcomes of personalized interventions, or contextualize data obtained in clinical settings, makes them a new and important complement to traditional clinical trials and established forms of medical knowledge production (Wicks et al. 2011; Swan 2012; Jain et al. 2015; Ritter 2015).

While the digitalization of medical research is largely institutionally driven, at the level of individual patients and citizens, the abundance of data that tracking devices can generate also promises to transform the relationship of individuals to their own health and further to their bodies, their psyches, and their selves. In many advertisements and popular accounts of self-tracking, the notion that collecting fine-grained details about various aspects of one's body can lead to unique insights about oneself is key. Nowhere is this quest for personal meaning about oneself via self-tracking more pronounced than in what is known as the Quantified Self movement. The Quantified Self was founded in 2007 by Gary Wolf and Kevin Kelly, two editors of Wired magazine, who felt that the rise of self-tracking in their San Francisco environment deserved a website and a place where enthusiasts could meet, share their self-tracking experiences, and discuss their findings. Since then, the group has expanded internationally and currently includes over 60,000 members across the globe, organized in over 200 groups that hold regular meetings, as well as two international conferences a year.

The explicit motto of the Quantified Self movement, branded as a tag line on its official website, is "self-knowledge through numbers." References to the Delphic maxim to "Know thyself" are rife in the quantified self blogosphere (Wolf 2009; de Groot 2012) and presentations. Collecting data about oneself using digital devices, as it is often proclaimed, is an important route to understanding one's body and oneself (Ruckenstein 2014), allowing one to know, rather than guess, how and who one is; if one is healthy, if one is productive, and if one is happy.

Larry Smarr, a well-known member of the Quantified Self who discovered he had Crohn's disease before his doctors thanks to his extensive self-tracking, outspokenly maintains that one cannot trust how one "feels" and that certainty lies only in what can "actually" be measured. This notion, that human perception is prone to bias and that subjective experience, intuition, and haptic senses are somehow lacking in comparison to the arithmetic precision of quantification, lies at the core of the quantified pursuit of self-awareness and self-knowledge. As a blogger writes, "Quantified self gives you a clear picture of yourself ... Selftracking tools and platforms provide us with accurate quantified feedback on

\footnotetext{
${ }^{3}$ Currently, more than a dozen studies are being carried out using the ResearchKit by major research institutes including Duke, John Hopkins, Stanford, and Oxford, in cardiovascular disease, Parkinson's, asthma, diabetes, and other conditions (cf http://www.imedicalapps.com/)
} 
ourselves. It's like facing a mirror" (Abrugar 2014). The image of a mirror, in opposition to a window, is one that Gary Wolf himself has introduced to explain what the Quantified Self movement is about. The data generated by self-tracking, as Wolf has explained on various occasions, does not open a window onto some deep inner truth but acts as a mirror to the mundane, everyday self that is made up of the habits, unconscious actions, and unperceivable patterns which we normally do not, or cannot, take notice of. In the New York Times cover article that he penned, which acts as somewhat of a manifesto for the movement, Wolf writes,

When we quantify ourselves, there isn't the imperative to see through our daily existence into a truth buried at a deeper level. Instead, the self of our most trivial thoughts and actions, the self that, without technical help, we might barely notice or recall, is understood as the self we ought to get to know. (2010)

It is precisely this trust in numerical data and what is deemed an excessive valuation of quantified knowledge that many critics of self-tracking and the Quantified Self movement have targeted in the media and academic literature, where self-quantifiers are commonly dubbed "data-obsessed," "data sexuals," "data fetishists," and "data junkies” (Basulto 2012; Morozov 2013; Dormehl 2014; Rettner 2014). The core of this critique is the idea that quantification and digitization are, in themselves, inherently reductionist processes (see also Sharon and Zandbergen 2016). Self-tracking works on the basis of categories or indicators that act as proxies for what are commonly very messy and rich phenomena, from "mood" to "health" to "productivity." When devices are described as giving users a "dashboard" or a "perfect picture" of their health, these data have a tendency to come to denote what health is. Similarly, explain critics, the relationship of variables commonly tracked in diet apps to weight gain and weight loss, and to health in general, is not something that can be reduced to a simple algorithm (Dunn 2013; Scrinis 2013). Measuring calories and carbohydrates is not tantamount to measuring nutrition, just as "actionless sleep" is not the same as "good sleep" (Paquet et al. 2007). While these variables can be neatly conveyed in numbers, these are numbers that have been extracted from their broader and more disorderly metabolic, environmental, and cultural contexts and then come to seamlessly represent the definitive truth about what healthy food, healthy sleep, and healthy living mean. The claim here is not just that the scientific accuracy and objectivity of self-tracking endeavors are questionable. More importantly, it is the reductionist effect of self-tracking practices on the richness and complexity of human experiences that is disputed.

Furthermore, as self-tracking seeks to reduce phenomena to numbers, critics argue that this simultaneously displaces other, non-quantifiable yet highly insightful means of knowing and expression. Lupton (2013), for example, argues that digital mobile technologies can be seen as a logical extension of the increasing use of visualization technologies in medicine that transform how the body and health are understood and represented:

Where once people relied upon the haptic sensations they felt in their bodies and reported to their physicians, medical technologies devoted to producing images of the body have altered the experience and treatment of bodies. The 
optic has come to take pre-eminence over the haptic in revealing the "truth" of the body. (2013, p. 6)

Critics are concerned that visual data will, by virtue of what Morozov calls its "imperialistic streak," overshadow other meaningful aspects of our lives. "What this stuff steals is our liveliness," a concerned novelist says in a New York Times article on the omnipresence of data and tracking devices, "Grids, spreadsheets and algorithms take away the sensory connection to our lives ... all the raw materials of life" (in Feiler 2014). As one's trust in numbers grows, it is feared, one's trust in subjective, embodied, and intuitive knowledge decreases.

This reductionist critique is supplemented by the notion that an excessive trust in numbers also obscures the fact that there is always a politics of measurement and that numbers are never, as they might seem to be, entirely neutral and impartial. Algorithms and big data, as critical data theorists argue, are anything but "raw" and objective (Gitelman 2013). They are shaped by social worlds, rife with hidden biases and embedded value judgments (boyd and Crawford 2012; Andrejevic 2014). In terms of self-tracking, this means that these devices configure ideals of "wellness," health, and "happiness" in ways that reproduce normative stereotypes, thereby pushing users to think about their own behaviors in accordance with predetermined standards and to conform to them in practice. Lupton (2015) discusses how a host of new apps aimed at quantifying users' sexual and reproductive activities and functions reinforce normative ideas of what "good sex" is and reproduce gender stereotypes as they encourage users to quantify their sexual experiences:

The discourses of performance, quantification and normality suggest specific limited types of sexualities. Gender stereotypes are reinforced by the focus on male performance (quantifying thrusts and duration of intercourse) and comparing sexual achievements (number of sexual partners, how often sex takes place, the quality of the experience). (2015, p. 8)

The excessive belief in the objectivity of numbers, in other words, risks causing what Morozov calls a "flight from thinking." For him, "One of the biggest dangers of the Quantified Self movement is that, in their belief in the natural origins of data, adherents will not question - or even reflect upon - the appropriateness of the measurement schemes that underwrite their data-gathering efforts" (2013, p. 246).

Furthermore, as described previously in relation to self-tracking and cultures of surveillance, the personal data that is generated by users of self-tracking technologies can, in a process of "function creep" (Whitson 2013), be employed by various actors for purposes that users are not aware of. For some theorists, this marks a shift to a somewhat more subtle yet no less pervasive form of social control and regulation, via the digital constructions of "new algorithmic identities" (Cheney-Lippold 2011) that can be used to exclude certain individuals and groups from access to goods and services or to identify them as security risks. Rather than resulting in greater self-knowledge then, self-tracking in this context is seen as contributing to both reductionist understandings of health and selfhood and new avenues for algorithmic intervention and control of people's lives, both of which can actually distance people from their "true" selves. 


\section{Moving Beyond a Polarized Debate}

In summary, current discussions surrounding the use of self-tracking technologies in the move toward PHC can be depicted in terms of three key polarizations: empowerment vs. discipline and surveillance, individual vs. collective responsibility for health, and greater self-knowledge vs. alienation via quantification. As I hope to have spelled out, many of the arguments made for and against the desirability of self-tracking for health in these discussions are persuasive. They also, however, present important limitations that should be made explicit and grappled with if we want to understand how people are experiencing the use of self-tracking for health in the move toward PHC.

\subsection{Of Values and Practices}

First amongst these limitations is that many of these claims are taking place in a highly theoretical and even speculative vacuum, certainly in the case of the promises being made, but also, often, in the case of the anticipated fears. In other words, a number of the assumptions underlying these claims need further empirical examination. As shall be discussed in more detail below, some of the important questions that emerge from this debate are, to an important extent, empirical questions that can be addressed using empirical, namely ethnographic methods. Another limitation is that the promises put forward, especially concerning increased empowerment by means of individual responsibility, emerge as highly problematic, if not empty labels used for predominantly economic interests, as the critique of those promises makes clear. At the same time, concerns of discipline, digital labor, and of individuals voluntarily partaking in selfsurveillance run the risk of configuring users of self-tracking devices as mindless dupes, unaware of or uninterested in the political and economic agendas at play. This is to say that on both sides of this debate, users of self-tracking devices are often configured in unrealistic ways: either as rational consumers who make autonomous health choices or as victims of insidious forces to which they remain oblivious. Lastly, this debate is not at all restricted to the question of the impact of self-tracking and PHC on health outcomes. Rather, these promises and concerns coalesce around three fundamental values that are at stake in the move toward PHC and the widespread adoption of self-tracking for health, to which both sides of this debate appeal - the values of autonomy, solidarity, and authenticity.

The promise of empowerment that has been shown to be key in the PHC and digital health debate thus invokes, in various ways, the value of autonomy. Self-tracking devices are envisaged here as enhancing the autonomy of patients and citizens: in relation to dependencies on others, in the form of the healthcare system or family caregivers (self-monitoring for independent living), in relation to medical professionals (informed patients participating in clinical decision making), and even in relation to unpredictable, naturally occurring processes (taking control over a disease and managing risk). Against this promise, critics raise concerns that PHC and self-tracking for health will be disempowering, by heralding a new age of pervasive health (self-)surveillance, and because self-tracking may soon become an obligation imposed 
by employers, insurers, and societal pressures, more than a voluntary choice. These critics are also appealing to the value of autonomy (Sharon 2015). ${ }^{4}$ Here, discourses of empowerment and "healthy citizenship" are seen as concealing economic realities that are often detached from the interests of citizens and patients and of creating new forms of discipline, subjection, and social control — of imposing limits on the autonomy of individuals. As Harris et al. (2010: 212) write: "empowerment discourses may be used to constitute citizens' choices in ways that increasingly subject them to external power, rather than enabling them to exercise their own."

Similarly, in the second polarization, both critics and advocates appeal to the value of solidarity. The critique of digital and personalized healthcare, as explained, and of the new emphasis on personal responsibility for health and welfare in general, is usually framed in terms of a conflict between neoliberal ideology and social solidarity_-"Me" vs. "We" in Dickenson's terms - in which the decline of solidarity is often deplored. As per Zygmunt Bauman, while individualization, privatization of risk, and uncertainty are on the rise, the "spirit of solidarity is in exile" (Bauman 2013). Advocates of self-tracking, on the other hand, also appeal to the value of solidarity. Here, citizens are encouraged to take personal responsibility in view of solidaristic objectives: in order to prevent the overburdening of the system. Terms like "limits to care" or negative references to "luxury medicine" and "medicine on demand" are used to justify cuts in healthcare services (ter Meulen 2000), and discourses of efficiency are cast as a means (not an end) in healthcare reforms that we must all carry.

Likewise, in the discussion around the types of knowledge that can be produced using self-tracking devices, both advocates and critics appeal to the value of authenticity, in the form of an enhanced or an impoverished relationship to one's self. On the one hand, self-tracking for health heralds the promise of greater and more precise medical knowledge, which will contribute to generating richer and more holistic representations of individual patients. At the level of individual users, this promise is construed as the transformation of one's relationship to one's health and further to one's body, psyche, and self, based on data that can reflect back a truer or more authentic self, as the Quantified Self motto "self-knowledge through numbers" implies. Conversely, critics are wary that a growing infatuation with quantified data, reflected at both institutional-scientific and citizen-consumer levels, may alienate individuals from authentic experiences informed by embodied knowledge and intuition. These devices, holds this critique, work by reducing rich and complex phenomena like health, wellbeing, mood, and selfhood to categories and numbers that have been shaped by others (usually with normative and commercial interests in mind) and, via this reductive logic, may constrain and override alternative means of self-expression.

Such a rendering explicit of the values that are at stake in these discussions offers several advantages. It provides some clarity in an otherwise complex and convoluted debate and can tie it to broader socio-political transformations than just healthcare. Furthermore, it is a way of foregrounding what matters to people. And lastly, it shows that the diverse claims that are being made on either side of this discussion are not wholly incommensurable.

\footnotetext{
${ }^{4}$ Even though these critiques often draw on a Foucauldian approach to power that seeks to problematize notions of freedom and autonomy. For a discussion on how these critiques fall back into an explanatory framework of autonomy vs. heteronomy by interpreting discipline as the absence of freedom, see Sharon (2015).
} 
The limitations discussed here-insufficient empirical basis, unrealistic configuration of users and implicit values and normative assumptions - indicate that the debate on self-tracking for health is to an important extent detached from the actual, everyday experiences of users of self-tracking devices. One important means of addressing these limitations is to focus on self-tracking for health as a practice. Practice theories have become increasingly popular in recent years in philosophy, cultural theory, sociology, anthropology, and science and technology studies, as offering a new vista for understanding social reality (see, for example, Schatzki et al. 2001; Reckwitz 2002; Nicolini 2012). At the core of these approaches is the idea that the primary relation of humans to the world is not by way of contemplation and representation, but through practical activity. This understanding effectively roots phenomena like knowledge, meaning, and power in practices and maintains that these phenomena must be analyzed via the field of practices. Within such a practice-based approach, values are also conceptualized as emerging from within practices. Rather than abstract entities_-"Truth," "Freedom," "Friendship" - or universal truth claims that precede or transcend experience, values are understood here as embedded in and as taking shape in everyday practices. They are articulated in specific acts or "enacted" (Mol 2002; Pols 2012; Swierstra 2013). Such an approach goes some way in explaining why the attempt to define a value unambiguously for all cases in which it is at stake often runs into difficulties, just as it can help explain how two opposing sides of a debate can persuasively appeal to one and the same value, be it autonomy, solidarity, or authenticity. It implies that values have multiple meanings since, insofar as they are enacted, they can be enacted in different ways in different contexts.

A shift of emphasis toward practices and a reframing of the debate on PHC and selftracking in terms of values thus offers an alternative framework from within which to describe and begin to evaluate the use of self-tracking tools. In the remaining portion of this paper, I begin to sketch out what this might look like, based on observations and interviews carried out within the Quantified Self community of self-trackers at various meetings and international conferences between 2013 and 2015, where members demonstrate, describe, and discuss their self-tracking practices. I show how this approach can make visible alternative articulations or unexpected enactments of the values at stake in the debate and thus lay the groundwork for new conceptual interventions and future research. Here, for example, a practice-based approach can help formulate a new set of questions: How is autonomy enacted in self-tracking practices that partake in a culture of healthism and surveillance? How is solidarity enacted when it is based in affinities that are biological, rather than social, and shared by few rather than many? And, how is authenticity enacted when numbers are seen as the preferred route to self-knowledge?

\subsection{Enacting Autonomy}

The Quantified Self (QS) community is characterized by local meetings where members get together to share their first-hand experiences using self-tracking methods and tools in short show-and-tell presentations. Guided by three "prime questions" (what did you do?, how did you do it?, what did you learn?), these personal presentations range from topics like what someone learned from 30 days of continuous ECG monitoring, to the effects of tracking breath on stress levels. At the annual European conference of 
2014 (QSEU14), one participant, Denise, told the story of how some 2 years before, despite a relatively happy and care-free life, she started having problems with her sleep. Using a sleep tracking app, she began recording the effects of a number of variablestypes of food, eating times, social life, and emotional matters - on the quality of her sleep. At the same conference, Paul, always interested in understanding his reactions to situations of stress from his days as a combat soldier to office negotiations, described the intricate experiment that he devised to track his anxiety and negative emotional states, and the different emotional triggers associated with them. As the subject matter of these exposés show, self-tracking for members of the QS is often a form of selfexperimentation that is driven by the desire to discover something particular to themselves, as unique individuals; these are " $n=1$ " experiments.

The appeal to individual uniqueness in relation to health is certainly an important dimension of the PHC discourse, in its move away from a so-called one-size-fits-all approach to more targeted treatment. While in practice, this has been realized at the level of sub-populations rather than at the individual level per se (Tutton 2012), for QSers, the focus on the individual is taken very literally. Here, the idea that what is "good," "right," or "healthy" for one person differs for every individual is a fundamental axiom. The knowledge that QSers gain from their $n=1$ experiments is highly particularistic and idiosyncratic, as they ask "what is the most favorable bedtime for $m e$," "what is the diet regime that works best for me," and "what activities, nutriments, and environment correlate optimally in light of the conditions of $m y$ life?" In this context, what constitutes a healthy practice may be completely different for different people, not just as a deviation from a norm, but as something that actually challenges the very existence of a norm. While this emphasis on individual health and well-being does reproduce the disciplinary dimension of the discourse of healthism, as critics in the self-tracking and PHC debate point out, it simultaneously disputes the content of that discourse by subjecting it to (at least somewhat) scientific scrutiny and thus challenges authoritative health claims. This kind of empowerment "in the wild" differs significantly from the controlled or guided type of empowerment that public health agencies and medical professionals have in mind when promoting digital health, and it imbues the practices of self-quantifiers with a very strong sense of agency and autonomy.

In these self-experiments, QSers are constituted as subjects who do not fit categories (Nafus and Sherman 2014), for whom state-sponsored messages of health and industrydetermined variables and functions are often seen as ill-fitting or not fine-grained enough. Autonomy, here, is enacted by either hacking and dismantling tracking devices, something that a number of the tech-savvy members of QS enjoy doing or, more commonly, by defining and constituting their own tracking parameters and analytical categories. For example, the four parameters that Denise tracked in relation to her sleep were not pre-defined, but significant for her in light of her everyday life. The experiment that Paul set up was even less guided, insofar as he first logged his reactions to situations in a random way, using an app that allows users to configure and customize their own categories, and then explored correlations that emerged. Such selftracking practices, as Nafus and Sherman (2014) observe in their ethnography of the QS movement, can be characterized as a form of "soft resistance" to dominant modes of living with data, a resistance that is not necessarily explicitly oppositional, but that is informed by a strong sense of autonomy. In this sense, as a participant explained at QSEU14, an important distinction can be made between "tracking yourself" and 
"being tracked by others," between a "doctor putting you on a scale" and doing so yourself.

In an interview that took place during QSEU14, Gary Wolf, co-founder and leading figure of QS, also identified resistance as a key dimension of what self-tracking is about, distinguishing between a resistance of "working with" and a resistance of "being against": doctors are not enemies, he explains, but there is much that an individual can do to better their situation independently of the authority of others, and self-tracking, for Wolf, offers an important means of achieving this. One of the important questions that Wolf sees as underpinning the self-reflexive discussions of QS meetings is, indeed, "how do you imbue your practice of normalizing your experiences with a spirit of autonomy?" This spirit of autonomy comes to the fore as an attempt to navigate a complex world where various messages and social conventions conveyed by the media, by medical institutions, and by the state tell people what to do and to carve out a space of agency therein.

One QSer's project presented at QSEU14, to track her grief following the unexpected death of her mother, illustrates this. The project, which consisted of a custommade digital spreadsheet, where she could log various experiences related to her grief, including sights, conversations, and events that elicited memories of her mother, where they took place and the mood she associated with them, was not only a therapeutic labor of sorts. It was also the creation of a reflective space in which the user could come to terms with loss on her own terms, unhindered by the social conventions that govern the grieving process, such as expectations of how long it should take and how much one should be immersed in it. Thus, as she explained, she not only shunned the use of existing mood trackers and created her own mood categories ("They just didn't quite fit. Mood tracking seems to assume that you want to track away the sadness"). But, as she and other participants in a breakout session fleshed out, the project became a way for her to tangibly "make a space" for grief and loss and to protect it from "the outside," from a "world that doesn't make space for grief and loss."

A "spirit of autonomy," as Wolf calls it, also runs through many of the origin stories of the QS community as they are told and re-told at QS meetings, where tracking often takes on more explicit forms of challenging scientific and medical authority. Larry Smarr, mentioned above, who discovered that he had Crohn's disease despite doctors dismissing his concerns, is somewhat of a QS hero. His open defiance of his medical team through his continued self-tracking is often referred to in QS discussions in romantic terms. Similarly, Seth Roberts, a psychologist and self-quantifying pioneer well known in the community for his peculiar self-experiments, has been upheld as someone who, in his belief that there are other ways of doing science than the randomized control trial and that experts can be wrong, embodies the real spirit of scientific curiosity. Roberts passed away some weeks before QSEU14, and the conference's closing plenary was an emotional tribute to him, led by Wolf and during which others related anecdotes. Wolf eulogized Roberts, not so much for his contribution to scientific knowledge, but for his authenticity: for being disloyal to the professional, institutional version of science, for not conforming to scientific rituals, and for being "dubious about current views about the world."

This individualist, non-conformist dimension of QS narratives may not be surprising. As a movement that was founded in the San Francisco Bay Area, by editors of 
Wired magazine, QS draws on a rich historical tradition of high-tech counterculturism, in which digital technologies have often been configured as subversive tools of resistance in the hands of individuals (Turner 2006). ${ }^{5}$ Nevertheless, in the context of growing concerns around expansive surveillance practices by governments and corporations, QSers' continuous tinkering with the hardware and software of their tracking tools and the refusal of some to use proprietary software and data platforms that are owned by private companies enact a form of autonomy that constitutes them as active users, rather than passive consumers of technology. As Wolf explained in a personal conversation that took place at QSEU15, the deep-seated skepticism that characterizes the tracking practices of many individual QSers is not reserved for state organizations, but also directed at corporations. The development of viable, tangible solutions to issues of data management, data protection, and privacy concerns is consequently an important focus of discussions on the QS forum and at international conferences and meetings. Thus, far from demonstrating an uncritical relationship to data collection or an obliviousness of its uses for and by others, as critics of QS often suggest, posing critical questions and exploring best practices around these issues are a main preoccupation of the community.

\subsection{Enacting Solidarity}

The highly idiosyncratic and particularistic nature of the self-tracking experiments undertaken by QSers described here has been a source of antagonism in the popular media and in some academic literature dealing with QS. As a personal enterprise that leads to insights and benefits that focus on the individual practitioner, self-tracking is often perceived as a solipsistic and narcissistic practice (Morozov 2013; Lupton 2015). Moreover, insofar as self-tracking is seen as paradigmatic of the shift toward "Me Medicine" (Dickenson 2013), critics also see it as exemplary of the anti-solidaristic tendencies of PHC, as discussed. Yet, for members of QS, and arguably for many selftrackers beyond QS, a crucial aspect of self-tracking is the opportunity to share their data with others and to participate in self-tracking communities of various sorts. Unlike many earlier, non-digital forms of self-tracking, like diary keeping, weight charts, or analogue speedometers, digital self-tracking tools usually have built-in functions that enable and encourage users to share and compare their findings with others, via social media and specially created platforms. To be sure, the promotion of sharing practices is currently the focus of an extensive critical discussion, as has been spelled out in previous parts of this paper, namely concerning the exploitation of shared data for commercial profit and surveillance. But this critique should not obscure the strong communal character that self-tracking takes on in various contexts and emerging spaces, both online and offline, in which enactments of solidarity may be materializing.

The opportunity for QSers to meet and discuss their experiences is a fundamental aspect of the QS movement. In these gatherings, the show-and-tell format described above is the framework through which participants share their experiences, while data takes on the form of a "common language" that "everyone can relate to," as per one participant at QSEU14, that makes this possible. As a QS media artist explains at this

\footnotetext{
${ }^{5}$ For a discussion on how this resistance both feeds into and helps reshape mainstream technological culture, see Zandbergen (2011) and Sharon and Zandbergen (2016)
} 
meeting, tracking is a way of preserving fleeting memories as they were at the time that they were logged, not just for herself, but for other people to see. This is to say that the data that is generated by tracking devices is not just a tool for gaining insights about oneself but becomes a medium for connecting with others by offering a raw glimpse into one's intimate, private life (Sharon and Zandbergen 2016). This communicative and communal dimension of tracking challenges allegations of narcissism and navel-gazing.

Self-disclosure and self-confrontation are commonplace in QS gatherings and infuse these meetings with a confessional quality. "What do you do when your data shows you something that you really don't like about yourself?" asks one participant. Indeed, selftracking experiments typically focus on aspects of individuals' lives that are disturbing, painful, or embarrassing: struggles with weight loss, mental health and disease, bad habits, or undesirable character traits - sensitive issues that are not easily revealed in most contexts and that are given a voice via the abstract language of numbers. As one participant explained: "I could either say to people, 'I have a bladder problem', or I could say "I go to the toilet 12 times a day, how many times do other people go?" For many QSers, then, sharing tracking experiences becomes a means of reaching out to others who endure similar hardships, and of offering knowledge, support, and solace, while getting some in return. Thus, as the young woman who sought to track her grief explained, one of the benefits of her tracking project was that it allowed her not only to focus on her own grief, but also to teach grieving others what might work best. As Wolf writes: "We leave traces of ourselves with our numbers, like insects putting down a trail of pheromones, and in times of crisis, these signals can lead us to others who share our concerns and care enough to help" (2010). In this sense, rather than a group of dataobsessed narcissists, many QSers seem to be seeking to enter into dialogue with others with whom they share commonalities, namely vulnerabilities: to tragic events, to disease, and to undesirable habits. And, to the idea that although we are often held accountable as such - perhaps even in the community's motto itself - we rarely do act as rational agents, in full control of our own bodies and behaviors. The " $n=$ me" may effectively be transformed in such practices into an " $n=$ we." Insofar as these connections express a concern for others arising from a shared vulnerability, can they be seen as forming the basis for relations of solidarity or enactments of solidarity?

Prainsack and Buyx (2012: 346), who have recently argued for and developed a practice-based understanding of solidarity, define solidarity as "shared practices reflecting a collective commitment to carry 'costs' (financial, social, emotional, or otherwise)." It is difficult to say that the self-tracking practices discussed here straightforwardly convey a "commitment to carry costs," other than perhaps the immediate emotional and time-consuming cost involved in standing in front of a group and disclosing one's findings. Furthermore, one could argue if these practices do enact a form of solidarity, then this is a highly particularistic, or narrow, form of solidarity, which performs important exclusions as it delimits its community of membership: leaving out all those who are not part of the QS community, with its various socioeconomic advantages, or all those who do not share the vulnerability in question. Certainly, this does not align with the "contractual solidarity" (Houtepen and ter Meulen 2000) that has commonly acted as the foundation for social arrangements in contemporary European societies. However, these practices can effectively render similarities between people more visible-which may be conducive for solidarity (Walhof 2006; Prainsack 2014). Furthermore, the vulnerabilities addressed by self- 
trackers are also unspecific ones, potentially shared by all practitioners by virtue of their being human, in which case, self-tracking may promote an increased disposition to solidaristic relations that are more broadly inclusive and perhaps even universal. Finally, some tracking practices that community members partake in can be said to more clearly reflect a commitment to carry costs insofar as tracking is undertaken in ways and based on methods that intentionally seek to make the data that they generate valuable for others, often non-specific others.

At QSEU14, one of the breakout sessions was called "Can data make us more human?" Participants were asked: "How might we use our practices as a starting point from which to develop empathy for others? Can we transform our wealth of personal and experiential data into a platform for improving our connection to those around us and to the broader world?" Collective projects do seem to be one of the directions that some QSers will be taking in the future. The closing plenary on day 1 of QSEU14 was an attempt to come to terms with the quantified self "in a Quantified World." Here, one speaker presented a collective project called "Quantified Planet" that aims to combine individual self-tracking data with planetary environmental data for research on sustainable ecosystems. On this panel, another speaker made an emotional plea to the audience to focus their energies beyond their privileged selves and community toward others who are in need, asking how the QS community can impact human rights, poverty, and global health issues. One of the important discussions that has animated the QS community in the past years, moreover, has been the question of how self-tracking data can contribute to public health and, more specifically, the question of how to make self-experimentation a more valid and accepted form of scientific knowledge production. To this end, a number of members throughout the community frequently engage in group studies that pool together individual self-experiments, organized by platforms like "Genomera" or "Doubleblinded," or actively make their tracking data publicly available for researchers to use, for example, on the "Open Humans" network.

The leaders of QS have also begun to actively position themselves as supporters of the trend toward "catalyzing the use of personal data for the public good" (HDE 2016) and as mediators between stakeholders in the emerging landscape of public health research using personal health data. In 2014, QS Labs helped coordinate a symposium at the Qualcomm Institute at the University of California, San Diego, that brought together researchers, public health officials, self-trackers, and business and technology representatives on the topic of voluntary and participatory public health research. The "QS Public Health Symposium" has become an annual event that concurs with the research meetings of the Health Data Exploration Network. As Wolf explained during the 2015 meeting, until recently, QS has been successful in bringing together makers and users of self-tracking devices. It was now time, he believed, to bring government, research, and funding agencies into the picture. One of the first researchers to speak at the 2015 symposium set the stage by labeling self-generated health data "a new natural resource" that should be conceptualized as part of our commons, which can "benefit all of us, like clean air and clean water." Another speaker, one of several scientifically oriented self-trackers, passionately spoke about the need to make single-subject trials the new pinnacle of medical evidence. She described the efforts of the group of over 40 healthcare professionals that she is part of who regularly meet to discuss how to 
improve and expand single-subject research studies for public health. Humorously though with clear intent, a conversation instigated by Larry Smarr, one of the symposium's conveners, ensued about the creation of a journal for single-subject studies as a next step.

There is no question that claims to "contributing to the public good" that are systematically and often loosely attached to these self-tracking practices need to be critically scrutinized. Who is defining the public good, at what costs this contribution takes place, and if "the public" does indeed reap the greatest benefits from such initiatives, are questions that need to be explored. But the fact that they need to be asked does not suspend the solidaristic nature of these practices for practitioners, nor these practitioners' belief that their personal data is indeed a new natural resource that should be part of a commons. How to evaluate these enactments of solidarity and which norms make some solidaristic relations more valuable than others are questions that will need to be addressed in future research on mobile and personalized healthcare. Taking a practice-based approach to the study of values is a first step in this direction, insofar as it allows us to identify the emergence of new forms of solidarity in unexpected sites.

\subsection{Enacting Authenticity}

One could be forgiven for assuming that QSers suffer from a deep-seated obsession with data. The movement's motto, "self-knowledge through numbers," and statements made by leading QS members in talks and articles do often smack of an excessive belief in the objectivity and truthfulness of numbers. Wolf's seminal New York Times (2010) piece is titled "The Data-Driven Life." In the opening pages, he writes: "If you want to replace the vagaries of intuition with something more reliable, you first need to gather data. Once you know the facts, you can live by them." As discussed, critics argue that self-tracking can lead to reductionist understandings of rich and complex categories like health and selfhood, where numbers will be given priority over subjective, intuitive sensations. Rather than a gain in self-knowledge, or the development of a closer or more authentic relationship to one's self and one's body, critics claim that this may result in alienating practitioners from themselves. As Zandbergen and I have argued elsewhere (Sharon and Zandbergen 2016), however, the idea that QSers are enamored by the perceived power of truth and objectivity of data that they are data fetishists, is very limiting and fails to account for a variety of other ways that QSers render their tracking practices meaningful.

As we argue in that context, the relationship between so-called objective data and socalled subjective experience is hardly a zero-sum game for many QSers, but rather a tension and a negotiation that produces meaning, a process that QSers are often aware of partaking in. As one participant at QSEU14 explained concerning physical activity tracking, quite some "cross-checking" and "re-calibration" goes on between one's intuitions and what the data shows, and rather than a blind faith in the latter over and above the former, an attempt to align both ensues. Such "calibrations" are particularly interesting for thinking about how authenticity is enacted in a context where numbers are seen as a significant (if not a preferential) route to self-knowledge.

For many QSers, the data generated by self-tracking does not straightforwardly produce a definitive truth, a one-to-one representation of their health, life, or identity. Often, self-tracking data is referred to as a supplement, an additional layer of 
information that enhances or contributes to broader understandings and self-narratives. At QSEU14, one participant explained that quantified data are like "signals" that are used in a form of "digital storytelling," "mingled in" with one's story. The theme of storytelling is a recurrent one at QS meetings. "Telling stories is how we connect to each other", one breakout organizer at QSEU14 writes, "our data can be a rich repository of stories for ourselves, and the archives of human history." What data does then, in many cases, is provide a part of the story that is believed to have been previously inaccessible. One participant explained,

Speaking about yourself through the data you collect is another way of creating identity. It is sharing your identity through the lens of some sort of information about yourself. Whether or not it is microbiomic, your activity, [or] heartrate, it is another way of saying "I am someone and this is who I am."

Rather than a numerical and constraining understanding of the self, as critics decry, this may point to an open-ended aesthetic one, where identity is constructed and curated, the product of quantitative and qualitative piecings together- "quantifying yourself is like art," said one self-tracker at QSEU13, "constructing a kind of expression."

This curated and modulative understanding of selfhood and authenticity may not come as a surprise to those familiar with poststructuralist theories of subjectivity, in particular as they are applied to digital media. In his piece on transformations in the notion of personal authenticity induced by social media usage, for example, Rob Horning (2013) argues that authenticity no longer refers to some unique interior truth about the self, but to the synthesis of data created as a result of the exhortation to always measure ourselves and share that information. "I am what I track" is a statement that is commonly heard at QS gatherings, but there is much leeway as to what constitutes that "I"-and if both I's actually signify the same entity. In one plenary session at QSEU14, three different trackers described three different understandings of the self or relationships to their selves that were enabled by their tracking practices: an agonistic self, which emerged from conversations that one tracker had with "the self" that kept gaining weight; an empathetic or mindful self, which emerged from one tracker becoming more aware of his mundane everyday; and a fragmented self, which could be datafied, pulled apart, visually represented on cards "and then scattered," i.e., sold as art works for this tracker. However, it is not clear from listening to QSers that, as Honing argues, "the true self, from this point of view [...] emerges through information processing [...] algorithmic systems that report back to us the true nature of who we are."

Rather, for many QSers, while the self that is "reported back" from the algorithmic systems of tracking devices is taken be truthful to some extent, there is also an understanding that without context, data can only tell a partial story. This has led some QSers to begin speaking about a "qualified self" (Davis 2013) as a more accurate expression of the entanglements and negotiations between metrics and interpretive schemes that characterize the quest for self-knowledge. For example, in a blog post called "The qualified self: going beyond quantification," Eric Boam and Jarrett Webb contend that 
where the quantified self gives us raw numbers, the qualified self completes our understanding of those numbers ... with richer context, we can better understand the quality of these quantities, and thereby better understand our being. As this capacity advances, the emphasis shifts to more metaphysical ways of describing ourselves. (Boam and Webb 2014)

Importantly, Webb is also a technology designer working on the development of sensing technologies that he believes will do much more than current devices in terms of enriching self-narratives, possibly by using audio, olfactory, and taste mechanisms. In other words, for some trackers, numbers are not at all what self-tracking is really about. They may instead be more like an unsophisticated, intermediate stage toward more augmented senses and, perhaps, expanded notions of selfhood (Swan 2013). Kevin Kelly, co-founder with Gary Wolf of QS, has spoken of how the quantified self will in the future become an extended "exoself" (Kelly 2012), a self that is spatially expanded with a series of exosenses that would allow us to haptically feel the metrics generated by devices. One of the examples discussed by Kelly in this context is the "North Paw," a haptic compass that vibrates when the user is facing north, eventually training the user to feel the north. In these forms of self-tracking, tracking practices may activate a heightened awareness of one's body, self, and environment, while data may enable new types of self-expression and enactments of authenticity.

\section{Conclusion}

This paper argues that a shift of emphasis toward practices, and a reframing of the debate on self-tracking for health in terms of values, offers an alternative framework from within which to describe, examine, and begin to evaluate the use of self-tracking devices in the much anticipated move toward PHC. This is important because the debate on digital and personalized healthcare, as analyzed in this paper, involves several limitations. First, it remains highly speculative - certainly in terms of its promises, but also in terms of a number of its concerns. Second, it tends to configure users of self-tracking devices in unrealistic ways - either as rational consumers who make autonomous health choices or as victims of insidious political and economic agendas of which they are oblivious. Third, and in light of these, it is detached from the everyday experiences of people who are actually using self-tracking devices in their daily lives. A practice-based approach, I suggested, that studies how values are articulated or enacted in specific practices, is useful for moving beyond these limitations. It can lay the groundwork for a series of new conceptual interventions and questions, and it can reorient our focus to enactments of values in unexpected empirical sites.

This paper only began to sketch out which questions can be asked and what (re)articulations of the values of autonomy, solidarity, and authenticity may become visible in such an exercise. Many questions and other empirical sites need to be formulated and explored. The examples discussed here, to be sure, are drawn from a very specific group of practitioners, who share all the common characteristics known to "early adopters." This is part of what makes the Quantified Self such a fascinating site, particularly since many of the concerns raised by critics, about privacy, about who gets to interpret data and to what ends, are being discussed within the community. But it is 
also what makes the Quantified Self a limited site from which to draw any broader conclusions. Other empirical sites should include, for example, chronic patients, who may not take up self-tracking voluntarily, people who stop using self-tracking devices (a group that is growing), non-users, older people, who may be at a technological disadvantage yet make up an important target group, etc.

Importantly, new questions will emerge that can begin to address the normative import of these practices. For example, if I have highlighted that the value of autonomy is enacted differently in self-tracking practices on the backdrop of discourses of healthism and a culture of surveillance, we need to ask, as Nafus and Sherman (2014) do, what the difference between being situated in and adapting to certain discourses and uncritically reproducing them is and how to nurture that difference. If I have highlighted that self-tracking practices can open up new spaces for the enactment of solidarity, we need to ask what kinds of solidarities we want to foster. Not all enactments of values are good ones, nor or are all self-tracking practices. In other words, we should begin to ask, which self-tracking practices, in the context of different groups of practitioners, will enable enactments of values in such a way that they overcome both the promises and concerns in the debate on self-tracking and PHC? What kinds of practices, and under what conditions, will help secure the values of autonomy, solidarity, and authenticity in ways that are meaningful to individuals and collectives in the new networks of human actors, self-tracking technologies, and diverse expectations from and by citizens and patients that are taking shape? As all citizens become increasingly encouraged to practice self-tracking for health by various political, economic, and moral forces, and as we move toward more personalized healthcare, these questions should become a focus of future research.

Acknowledgments This research was supported by a grant from the Netherlands Organization for Scientific Research (grant number 275-20-050). I wish to thank the participants of the paper roast session at 'TILTing perspectives 2015', Gary Wolf and the many self-trackers who provided me with insights, as well as my anonymous referees for their valuable suggestions.

Open Access This article is distributed under the terms of the Creative Commons Attribution 4.0 International License (http://creativecommons.org/licenses/by/4.0/), which permits unrestricted use, distribution, and reproduction in any medium, provided you give appropriate credit to the original author(s) and the source, provide a link to the Creative Commons license, and indicate if changes were made.

\section{References}

ABI research (2013) Wearable computing devices, like Apple's iWatch, will exceed 485 million annual shipments by 2018. Retrieved from https://www.abiresearch.com/press/wearable-computing-devices-likeapples-iwatch-will. Accessed 25.2.2015.

Abrugar, V. (2014) Quantified self: how self-tracking technology can improve your life. http://blog.goalmap. com/quantified-self-how-self-tracking-technology-can-improve-your-life/. Accessed 13.3.15.

Andrejevic, M. (2014). The big data divide. International Journal of Communication, 8, 1673-1689.

Armstrong, D. (1995). The rise of surveillance medicine. Sociology of Health \& Illness, 17(3), 393-404.

Ayo, N. (2012). Understanding health promotion in a neoliberal climate and the making of health conscious citizens. Critical Public Health, 22(1), 99-105.

Basulto, D. (2012) Meet the urban datasexual. Retrieved from http://bigthink.com/endless-innovation/meetthe-urban-datasexual. Accessed 13.2.2015.

Bauman, Z. (2013). Solidarity: a word in search of flesh. Eurozine, May 8. http://www.eurozine.com/articles/ 2013-05-08-bauman-en.html. Accessed 16 Mar 2015. 
Boam, E. \& Webb, J. (2014) The qualified self: going beyond quantification. Retrieved from http:// designmind.frogdesign.com/2014/05/qualified-self-going-beyond-quantification/. Accessed 20.3.2015.

Boyd, D., \& Crawford, K. (2012). Critical questions for big data: provocations for a cultural, technological, and scholarly phenomenon. Information, Communication \& Society, 15(5), 662-679.

Burrows, R., Nettleton, S., \& Bunton, S. (1995). Sociology and health promotion: health, risk and consumption under late modernism. In R. Bunton, S. Nettleton, \& R. Burrows (Eds.), The sociology of health promotion: critical analyses of consumption, lifestyle and risk. New York: Routledge.

Byrne, D. \& Brand, H. (2013) Meeting the challenge: special supplement on personalized medicine. Parliament Magazine September.

Campbell, D. (2012) Doctors back denial of treatment for smokers and the obese. The Guardian http://www. theguardian.com/society/2012/apr/28/doctors-treatment-denial-smokers-obese.

Cesuroglo, T., et al. (2012). Public health perspective: from personalized medicine to personalized health. Personalized Medicine, 9(2), 115-119.

Cheney-Lippold, J. (2011). A new algorithmic identity: soft biopolitics and the modulation of control. Theory, Culture and Society 28(6), 164-181.

Clarke, J., Newman, J., Smith, N., Vidler, E., \& Westmarland, L. (2007). Creating citizen-consumers: changing publics and changing public services. London: Sage.

Coggi, P. T. (2011). A European view on the future of personalized medicine in the EU. European Journal of Public Health, 1, 6-7.

Crawford, R. (1980). Healthism and the medicalization of everyday life. International Journal of Health Services, 10(3), 365-388.

Crawford, R. (2006). Health as a meaningful social practice. Health, 10(4), 401-420.

Davis, J. (2013). The qualified self. Available at: https://thesocietypages.org/cyborgology/2013/03/13/thequalified-self/. Accessed 31 Mar 2016.

De Corde, C. \& Rolvink Couzy, F. (2015) Achmea geeft premiskroting aan klant die data levert. Finacieele Dagblad. http://www.expatica.com/nl/news/country-news/DN-Dutch-Insurance-giant-to-link-premiumdiscounts-to-customer-behaviour_509426.html. Accessed 2.2.2016.

De Groot, M. (2012) Knowledge is power, know thyself! Retrieved from http://www.qsinstitute.org/?p=2067. Accessed 20.3.2015.

Dhar, V. (2014). Healthcare and data: an interview with Peter Szolovits. Big Data, 2(4), 182-184.

Dickenson, D. (2013). Me medicine vs. We medicine: reclaiming biotechnology for the common good. New York: Columbia University Press.

Dormehl, L. (2014). The formula: how algorithms solve all our problems ... and create more. New York: Penguin.

Dunn, R. (2013) Science reveals why calorie counts are all wrong. Scientific American (April 20) 309(3). http:/www.scientificamerican.com/article/science-reveals-why-calorie-counts-are-all-wrong/?page=3. Accessed 11.2.15.

EAPM (European Alliance for Personalised Medicine) (2012) Personalised medicine: new perspectives for patients in Europe. http://www.phgen.eu/typo3/fileadmin/downloads/EAPM_Manifesto.pdf. Accessed 2. 19.2015.

EC (European Commission) (2012) eHealth action plan 2012-2020-innovative healthcare for the 21st century. Brussels.

EC (2013) Use of '-omics' technologies in the development of personalized medicine, working document

EC (2014a) GREEN PAPER on mobile health, COM (2014) 219. Brussels.

EC (2014b) COMMISSION STAFF WORKING DOCUMENT on the existing EU legal framework applicable to lifestyle and wellbeing apps SWD (2014) 135. Brussels.

EC (2014c) Healthcare in your pocket: unlocking the potentials of mHealth, IP/14/394. Brussels.

European Science Foundation (ESF) (2012). Personalized Medicine for the European Citizen - towards more precise medicine for the diagnosis, treatment and prevention of disease. Strasbourg: ESF. http://www.esf. org/index.php? e I D =tx_naw securedl\&u=0\&g=0\&t=1459438535\&hash= 46a03100e3aae993941fc0aa6cbd319d2492334f\&file=fileadmin/be_user/CEO_Unit/Forward_Look/ iPM/FL_2012_iPM.pdf. Accessed 30 Mar 2016.

Feiler, B. (2014) The United States of metrics. New York Times http://nyti.ms/1nXqSvP. Accessed 13.3.15.

Flores, M., et al. (2013). P4 medicine: how systems medicine will transform the healthcare sector and society. Personalized Medicine, 10(6), 565-576.

Foreman, A., et al. (2011). Just text me: using SMS technology for collaborative patient mood charting. Journal of Participatory Medicine, 3, e45.

Foucault, M. (1977). Discipline and punish: the birth of the prison (Translated by A. Sheridan). London: Allen Lane. 
Fox, S., \& Duggan, M. (2013). Tracking for health. Washington, DC: Pew Research Center. Gitelman, L. (2013). "Raw data" is an oxymoron. Boston: MIT Press.

Groves, P., Kayyali, B., Knott, D., Van Kuiken, S. (2013) The 'Big Data' revolution in healthcare: accelerating value and innovation. Report published by the Center for US Health System Reform.

Harris, R., Wathen, N., \& Wyatt, S. (2010). Configuring health consumers: health work and the imperative of personal responsibility. New York: Palgrave.

Harrison, V., et al. (2011). Mobile mental health: review of the emerging field and proof of concept study. Journal of Mental Health, 20(6), 509-524.

Health Data Exploration Network (2016). http://hdexplore.calit2.net/. Accessed 31 Mar 2016.

Hill, K. (2011) Adventures in self-surveillance, aka the quantified self, aka extreme navel-gazing. Forbes, Jul. $4 \mathrm{http} / /$ www.forbes.com/sites/kashmirhill/2011/04/07/adventures-in-self-surveillance-aka-the-quantifiedself-aka-extreme-navel-gazing/ Accessed 6.3.2015.

Horning, R. (2013) Google alert for the soul. The New Inquiry. http://thenewinquiry.com/essays/google-alertfor-the-soul/ Accessed 26.2.2016.

Houtepen, R., \& ter Meulen, R. (2000). New types of solidarity in the European welfare state. Healthcare Analysis, 8(4), 329-340.

Jain, S., et al. (2015). The digital phenotype. Nature Biotechnology, 33(5), 462-463.

Kelly, K. (2012) The quantified century. Retrieved from http://quantifiedself.com/conference/Palo-Alto-2012.

Laakso, E., Armstrong, K., \& User, W. (2011). Cyber-management of people with chronic disease: a potential solution to eHealth challenges. Health Education Journal, 5(3), 1-8.

Lupton, D. (2012). M-health and health promotion: the digital cyborg and surveillance society. Social Theory \& Health, 10(3), 229-244.

Lupton, D. (2013). Quantifying the body: monitoring and measuring health in the age of mHealth technologies. Critical Public Health, 23(August), 393-403.

Lupton, D. (2014) Self-tracking modes: reflexive self-monitoring and data practices. In Immanent Citizenships workshop, Canberra, AU, 27 August. Unpublished paper.

Lupton, D. (2015). Quantified sex: a critical analysis of sexual and reproductive self-tracking using apps. Culture, Health and Sexuality, 17(4), 1-14.

McGregor, J. (2014) Fitness trackers chase after the corporate market. The Washington Post. http://www. washingtonpost.com/blogs/on-leadership/wp/2014/12/18/fitness-trackers-chase-after-the-corporatemarket/. Accessed 3.3.2015.

Mol, A. (2002). The body multiple: ontology in medical practice. Durham: Duke University Press.

Morozov, E. (2013). To Save Everything Click Here. London: Allen Lane

Nafus, D., \& Sherman, J. (2014). This one does not go up to 11: the quantified self movement as an alternative big data practice. International Journal of Communication, 8, 1784-1794.

National Academy of Sciences (NAS). (2011). Toward precision medicine: building a knowledge network for biomedical research and a new taxonomy of disease. Washington, DC: NAS.

NHS Department of Health (2011) Innovation health and wealth, accelerating adoption and diffusion in the NHS. Retrieved from http:/webarchive.nationalarchives.gov.uk/20130107105354/http:/www.dh.gov.uk/ prod_consum_dh/groups/dh_digitalassets/documents/digitalasset/dh_134597.pdf. Accessed 26 Feb 2015.

NHS (2015). Technology enable $\bar{d}$ care services. NHS commissioning assembly. https://www.england.nhs.uk/ ourwork/qual-clin-lead/tecs/. Accessed 23.3.2015.

Nicolini, D. (2012). Practice theory, work, and organization. Oxford: Oxford University Press.

Norris, J. (2012) Self-tracking may become key element of personalized medicine. UCSF News http://www. ucsf.edu/news/2012/10/12913/self-tracking-may-become-key-element-personalized-medicine. Accessed 18.02.2015.

Olson, P. (2014a) Wearable tech is plugging into health insurance. Forbes. http://www.forbes.com/sites/ parmyolson/2014/06/19/wearable-tech-health-insurance/. Accessed 03.03.2015.

Olson, P. (2014b) The quantified other: Nest and Fitbit chase a lucrative side business. Forbes. http://www. forbes.com/sites/parmyolson/2014/04/17/the-quantified-other-nest-and-fitbit-chase-a-lucrative-sidebusiness/. Accessed 03.03.2015.

Paquet, J., Kawinska, A., \& Carrier, J. (2007). Wake detection capacity of actigraphy during sleep. Sleep, $30(10), 1362-1369$.

Pols, J. (2012). Care at a distance: on the closeness of technology. Amsterdam: Amsterdam University Press.

Prainsack, B. (2014). Personhood and solidarity: what kind of personalized medicine do we want? Personalized Medicine, 11(7), 651-657.

Prainsack, B., \& Buyx, A. (2012). Solidarity in contemporary bioethics - towards a new approach. Bioethics, 26(7), 343-350. 
Prainsack, B., \& Vayena, E. (2013). Beyond the clinic: 'direct-to-consumer' genomic profiling services and pharmacogenomics. Pharmacogenomics, 14(4), 403-412.

Rabin, C., \& Bock, R. (2011). Desired features of smartphone applications promoting physical activity. Telemedicine and e-Health, 17(10), 801-803.

Reckwitz, A. (2002). Towards a theory of social practices: a development in culturalist theorizing. European Journal of Social Theory, 5(2), 243-263.

Research2Guidance (2013) Mobile health market report, 2013-2017. http:/www.research2guidance.com/ shop/index.php/downloadable/download/sample/sample_id/262/.

Rettner, R. (2014) Quantified self movement: how data-obsessed self-trackers push toward healthier lives. Live Science. http://www.livescience.com/44618-quantified-self-culture.html. Accessed 30.04.2015.

Ritter, S. (2015). Apple's research kit development framework for Iphone apps enables innovative approaches to medical research data collection. Clinical Trials, 5(2), e120.

Rose, N. (1999). Powers of freedom: reframing political thought. Cambridge: CUP.

Ruckenstein, M. (2014). Visualized and interacted life: personal analytics and engagements with data doubles. Societies, 4, 68-84.

Schatzki, T., Knorr Cetina, K., \& von Savigny, E. (2001). The practice turn in contemporary theory. London: Routledge.

Scrinis, G. (2013). Nutritionism: the science and politics of dietary advice. New York: Columbia University Press.

Sharon, T. (2015). Healthy citizenship beyond autonomy and discipline: tactical engagements with genetic testing. Biosocieties, 10(3), 295-316.

Sharon, T. \& Zandbergen, D. (2016). From data fetishism to quantifying selves: self-tracking practices and the other values of data. New Media \& Society. doi:10.1177/1461444816636090.

Soreon Research (2014). The wearable health revolution: how smart wearables disrupt the healthcare sector. Switzerland.

Steinhubl, S., Muse, E., \& Topol, E. (2013). Can mobile health technologies transform health care? JAMA, $310(22), 2395-2396$.

Swan, M. (2009). Emerging patient-driven health care models: an examination of health social networks, consumer personalized medicine and quantified self-tracking. International Journal of Environmental Research and Public Health, 6, 492-525.

Swan, M. (2012). Health 2050: the realization of personalized medicine through crowdsourcing, the quantified self, and the participatory biocitizen. Journal of Personalized Medicine, 2, 93-118.

Swan, M. (2013). The quantified self: fundamental disruption in big data science and biological discovery. Big Data, 2(1), 85-98.

Swierstra, T. (2013). Nanotechnology and techno-moral change. Ethics \& Politics, 15(1), 200-219.

ter Meulen, R. (2000). The expectation(s) of solidarity: matters of justice, responsibility and identity in the reconstruction of the health care system. Health Care Analysis, 8, 355-376.

ter Meulen, R., \& Jotterand, F. (2008). Individual responsibility and solidarity in European health care. Journal of Medicine and Philosophy, 33(3), 191-197.

Terranova, T. (2000). Free labor: producing culture for the digital economy. Social Text, 18(2), 33-58.

Till, C. (2014). Exercise as labour: quantified self and the transformation of exercise into labour. Societies, $4(3), 446-462$.

Topol, E. (2015). The patient will see you now: the future of medicine is in your hands. New York: Basic Books.

Traynor, M. (2003). A brief history of empowerment: response to discussion with Julianne Cheek. Primary Health Care Research and Development, 4, 129-136.

Turner, F. (2006). From counterculture to cyberculture. Stewart Brand, the whole earth network, and the rise of digital utopianism. Chicago: University of Chicago Press.

Tutton, R. (2012). Personalized medicine: futures present and past. Social Science and Medicine, 75(10), $1721-1728$.

Walhof, D. (2006). Friendship, otherness, and Gadamer's politics of solidarity. Political Theory, 34(5), 569593.

Weber, G., et al. (2014). Finding the missing link for big biomedical data. JAMA, 311(24), 1-2.

Whitson, J. (2013). Gaming the quantified self. Surveillance \& Society, 11(1/2), 163-176.

Wicks, et al. (2011). Accelerated clinical discovery using self-reported patient data collected online and a patient-matching algorithm. Nature Biotechnology, 29(5), 411-414.

Wiederhold, B. (2012). Self-tracking: better medicine through pattern recognition. Cyberpsychology, Behavior and Social Networking, 15(5), 235-236. 
Wolf, G. (2009) Know thyself: tracking every facet of life, from sleep to mood to pain, 24/7/365. Wired, June $22 \mathrm{http} / /$ archive.wired.com/medtech/health/magazine/17-07/lbnp_knowthyself?currentPage=all. Accessed 20.3.2015.

Wolf, G. (2010) The data-driven life. The New York Times Magazine, April 28. http://www.nytimes.com/2010/ 05/02/magazine/02self-measurement-t.html?pagewanted=all\& r=0.

Zandbergen D. (2011) New edge. Technology and spirituality in the San Francisco Bay area. PhD dissertation. 